\title{
Hyperspectral and Multispectral Reflectance Imaging of Paintings
}

Pamela Betts ${ }^{1}$, Nicholas Barbi ${ }^{2}$, Glenn Gates ${ }^{1}$, Erich Uffelman ${ }^{3}$, Howland Jones ${ }^{4}$ and Gabor Kemeny ${ }^{5}$

${ }^{1}$ The Walters Art Museum, Baltimore, Maryland, United States, ${ }^{2}$ nSynergies, Inc, Sarasota, Florida, United States, ${ }^{3}$ Washington and Lee University, Lexington, Virginia, United States, ${ }^{4}$ HyperImage Solutions, Rio Rancho, New Mexico, United States, ${ }^{5}$ Middleton Spectral Vision, Middleton, Wisconsin, United States

Hyperspectral infrared reflectance Imaging (HSI) imaging has been increasingly applied to the study of paintings in recent years ${ }^{1-4}$. HSI provides images which may reveal such features as underdrawings, underlying paintings and pigment differences, and, by means of a spectrograph between the lens and the sensor, also provides a spectrum at each point in the infrared reflectance (IRR) Image. The spectral region covered by HSI depends on the camera: a camera using a silicon-based sensor covers both the visible light region (400-750 $\mathrm{nm}$ ) and the near infrared (NIR) region from 750-1000 nm (together, the regions are referred to as VNIR); a camera with an indium gallium arsenide (InGaAs) sensor can cover the range from 900-1700 nm; and a camera using a cooled HgCdTe (mercury cadmium telluride, or "MCT") covers from 1000-2500 nm (short wave infrared, or SWIR). InGaAs cameras, therefore, only partially cover the SWIR range. The MCT camera covers the longer wavelengths in the SWIR range, a region rich in spectral information, but adds expense and weight to the system

An example of HSI imaging of oil painting by Larry Elliott is shown in Figure 1. Figure 1a is a photograph (RGB, 400-750nm) of the painting, while the false color HSI image is shown in Figure 1b. The imaging was performed using an HSI system optimized for Cultural Heritage $(\mathrm{CH})$ applications, manufactured by Middleton Spectral Vision and incorporating a Specim SWIR3 MCT camera (384 spatial bands and 288 spectral bands). The analysis software, KemoQuant TM, performs both Principal Component Analysis $(\mathrm{PCA})^{5}$ and Multivariate Curve Resolution (MCR) ${ }^{6}$. The PCA result shown in Figure 1b shows both dramatic and subtle differences, some highlighted in red, which lead to better understanding of the artist's methods as well as the history of the painting.

An alternative to HSI, at the expense of high spectral resolution, is to record individual images using a high quality InGaAs camera with a series of filters in front of the lens, each covering a narrow band of wavelengths. This approach is called infrared "multispectral imaging" (MSI) reflectography". It should be considered when laboratories already have a high quality InGaAs camera and wish to add some spectral specificity to their imaging tool kit. Compared to HSI, MSI offers the advantage of higher image resolution because of the absence of the spectrograph in the image path and the availability of higher resolution cameras, but requires many repetitive images to be taken and always leaves spectral gaps no matter how many filters are used. The Walters Art Museum has an HSI system for the VNIR region, and an enhanced MSI system for the SWIR region to $1700 \mathrm{~nm}$. The enhancements to the MSI system are significant, adding 1) open source spectral Imaging Registration software from the National Gallery of Art (NGA) and George Washington Unveristy ${ }^{8}$, which registers the images from the many filters and mosaics multi and hyperspectral images, and 2) KemoQuant ${ }^{\mathrm{TM}}$ - $\mathrm{CH}$ software, which now allows the same PCA and MCR algorithms used in HSI to be applied to the discrete images resulting from filters (MSI). 

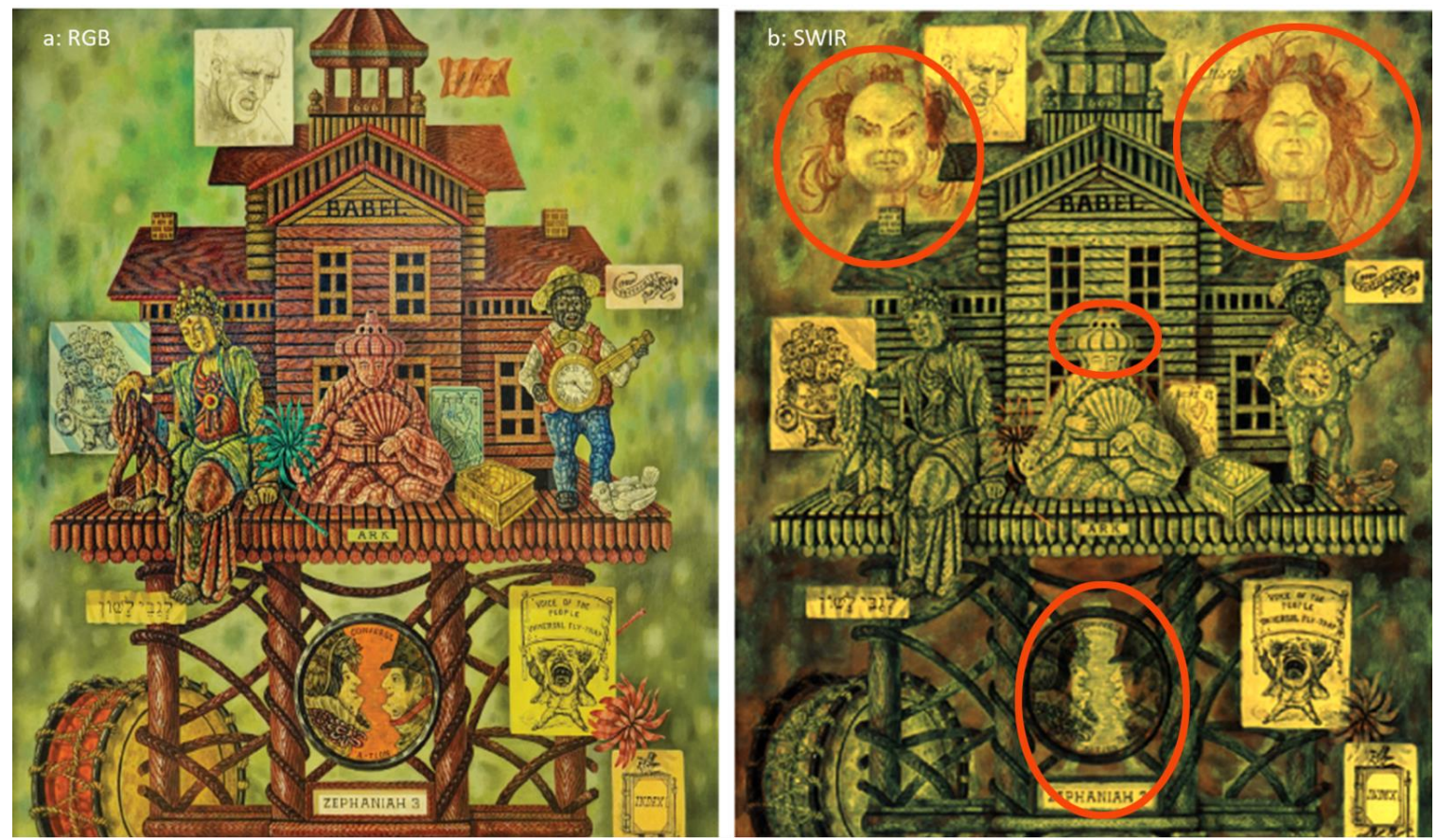

Figure 1. A Painting from the Estate of Larry Elliott. Artist: Larry Elliott (1948-2019). The Babbling Tower; Oil on panel; 24X20 in (61X51 cm); Catalog \#E-60/3461. RGB Photo is on left. SWIR HSI using Reveal Scan System (right) shows features, some highlighted in red, from an earlier version of the painting.
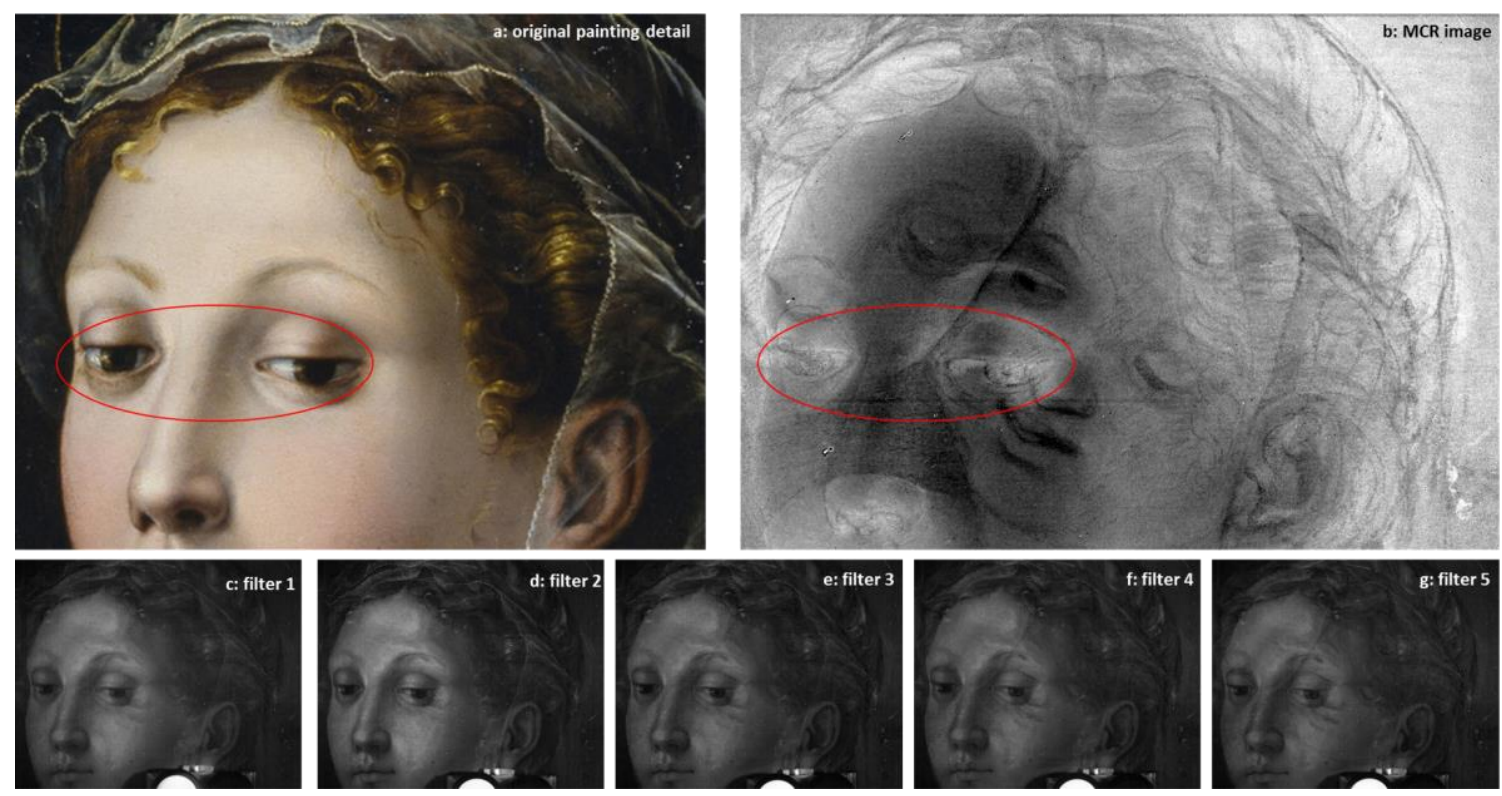

Figure 2. A Detail of a Painting in the Walters Collection attributed to Giulio Romano (c 1522-1524). The Madonna and Child with Saint John the Baptist, (37.548). Images c-g were recorded with an InGaAs camera using 5 filters. Underlying faces (b) are revealed after analysis in KemoQuant- $\mathrm{CH}$ software.

References

1. Cucci,C, Casini, A: Data Handling in Science and Technology, Volume 32, 2020, Pages 583-604 
2. de Manincor, N, Marchioro, G, Fiorin, E, Raffaelli, M, Salvadori, O, Daffara, C: Microchemical Journal, Volume 153, March 2020, 104469

3. Delaney, John K., Mathieu Thoury, Jason G. Zeibel, Paola Ricciardi, Kathryn M. Morales, and Kathryn A. Dooley. "Visible and Infrared Imaging Spectroscopy of Paintings and Improved Reflectography." Heritage Science 4, no. 1, 1-10 March (2016).

4. Huang, X; Uffelman, E; Cossairt, O; Walton, M; Katsaggelos, A: IEEE Signal Processing Magazine, Volume: 33, Issue: 5, Sept. 2016

5. Delaney, John K., Giorgio Trumpy, Marie Didier, Paola Ricciardi and Kathryn A. Dooley, “A High Sensitivity, Low Noise and High Spatial Resolution Multi-Band Infrared Reflectography Camera For the Study of Paintings and Works on Paper", Herit Sci 5, 32 (2017).

6. Conover, Damon M., John K. Delaney, and Murray H. Loew. "Automatic Registration and Mosaicking of Technical Images of Old Master Paintings." Applied Physics A 119, no. 4 (2015): 1567-1575.

7. Jones,H.D.T. et al. "Weighting hyperspectral image for improved multivariate curve resolution", Chemometrics 2008; 22: 482-490. [8] Conover, Damon M., John K. Delaney, and Murray H. Loew. "Automatic Registration and Mosaicking of Technical Images of Old Master Paintings." Applied Physics A 119, no. 4 (2015): 1567-1575. 